\title{
The Powdered Magnets Technology Improvement by Biencapsulation Method and its Effect on Mechanical Properties
}

Dorota Klimecka-Tatar

Institute of Production Engineering, Faculty of Management, Częstochowa Univesity of Technology, Armii Krajowej 19B, 42-201 Czestochowa, Poland. E-mail: klimt@wip.pcz.pl.

In this paper the technological process of bonded magnets manufacturing was described. The greatest dangers arising during the process steps that have a significant impact on magnetic, chemical and thermodynamic stability $\mathrm{Nd}-\mathrm{Fe}-\mathrm{B}$ bonded type of magnetic materials were also indicated. The effect of the biencapsulation of $\mathrm{Nd}_{12} \mathrm{Fe}_{77} \mathrm{Cos}_{5} \mathrm{~B}_{6}$ powder particles with $\mathrm{Ni}-\mathrm{P} / \mathrm{epoxy}$ resin, phosphate/epoxy resin and $\mathrm{Cu} / \mathrm{epoxy}$ resin layers on the finale magnets has been evaluated. The production processes in technological terms in original and after improving modification were presented - the technological process taking into account the stage of powder surface etching and the powder particles biencapsulation. It was proved that the preliminary biencapsulation of particles surface before consolidation in some cases significantly improve the mechanical properties of the bonded with epoxy-resin magnets.

Keywords: bonded magnets, biencapsulation, $\mathrm{Nd}-\mathrm{Fe}-\mathrm{B}$ magnets, magnetic composites

\section{Acknowledgments}

This research was supported by funds for education in the years 2010-1013 by Ministry of Science and Higher Education (Republic of Poland) as a research project No. N N507 616838

\section{References}

[1] BALA H., SZYMURA S. (1995) Inż. Materiałowa, 3, pp.119.

[2] BALA H., TREPAK N.M., SZYMURA S., LUKIN A.A., GAUDYN V.A., ISAICHEVA L.A., PAWŁOWSKA G., ILINA L.A. (2001). Intermetallics, 9, pp. 515.

[3] DIAN M. (2013). The Methodology of Quality Matrix in Manufacturing Quality Process Improvement. Manufacturing Technology. Vol. 13, No. 4, pp. 431-437.

[4] DOBRZAŃSKI L.A., DRAK M., TRZASKA J. (2005). J.Mater.Proc.Tech., 164, pp. 795

[5] EDGLEY D. S., LE BRETON J.M., STEYAERT S., AHMED F.M., HARRIS I.R., TEILLET J. (1997) J.Magn.Magn.Mater., 173, pp. 29.

[6] EL-MONEIMA A.A., GEBERT A., SCHNEIDER F., GUTFLEISH O., SHULTZ F. (2002), Corros.Sci. 44, pp. 1097.

[7] EL-MONEIMA A.A., GUTFLEISH O., PLOTNIKOV A., GEBERT A. (2002). J.Magn.Magn. Mater., 248, pp. 121.

[8] HARI KRISHNAN K, JOHN S, SRINIVASAN KN, PRAVEEN J, GANESAN M, KAVIMANI PM (2006) $M e$ tall Mater. Trans A 37.pp. 1917-1926.

[9] KLIMECKA-TATAR D, ŚLUSAREK B, BALA H, GĘSIARZ K (2005) Ochr Przed Koroz 11s/A, pp. 93-97.

[10] KLIMECKA-TATAR D, BALA H, ŚLUSAREK B, JAGIELSKA-WIADEREK K. (2009) Arch Metall Mater 54/1, pp. 247-256.

[11] KLIMECKA-TATAR D. (2011). The Production Process of Bonded Magnets Based on Microencapsulated Nd14.8Fe76Co4.95B4.25 Powder. Chapter 10. In:Toyotarity. Heijunka. Monography. Editing and Scientific Elaboration Stanisław Borkowski, Robert Ulewicz s.155-170 Dnipropetrovsk

[12] KLIMECKA-TATAR D, PAWŁOWSKA G, RADOMSKA K (2013) Ochr Przed Koroz, 56/5, pp. 187-190.

[13] PAWŁOWSKA G, KLIMECKA-TATAR D, MAZIK A (2013) Ochr Przed Koroz 56/4, pp. 174-176.

[14] KORDAS K, BEKESI J, VAJTAI R, NANAI L, LEPPAVUORI S, UUSIMAKI A, BALI K, GEORGE TF, GALBACS G, IGNACZ F, MOILANEN P (2001) Appl Surf Sci 172, pp. 178-189.

[15] LEONOWICZ M., WOJCIECHOWSKI S. (1988) Archiwum Nauki o Materiałach, 9, pp. 263.

[16] LEONOWICZ M., WYSŁOCKI J.J. (2005). Wspótczesne magnesy, WNT, Warszawa. 
[17] MAREK I., MICHALCOVA A., VOJTECH D. (2013). Properties of metallic nanocrystalline powders. Manufacturing Technology, Vol. 13, No. 3, pp. 353 - 358.

[18] RADA M., LYUBINA J., GEBERT A., GUTFLEISCH O., SCHULTZ L. (2005) J.Magn.Magn. Mater., 290, pp. 1251

[19] ŚLUSAREK B. (2001). Dielektromagnets Nd-Fe-B, Oficyna Wyd. Politechniki Wrocławskiej, Wrocław.

[20] SKULJ I., DOUVALIS A.P., HARRIS I.R. (2006). J.Alloy.Compd, 407, pp. 304

[21] ULEWICZ R. (2003). Quality control system in production of the castings from spheroid cast iron, Metalurgija Vol. 42, Issue 1, pp. 61-63.

[22] VALÁŠEK P., MÜLLER M. (2013). Polyurethane resins filled with inorganic waste particles. Manufacturing Technology. Vol. 13, No. 2, pp. 241-247.

[23] XIAO J., OTAIGBE J. (2000), J.Alloy.Compd, 309, pp. 100.

Copyright $($ ) 2014. Published by Manufacturing Technology. All rights reserved. 